\title{
Analysis of Bone Microarchitectural Changes and Structural Damage in Sickle Cell Disease-Induced Avascular Necrosis Using Raman Spectroscopy
} Is there potential for medical management?

"Ahmed Al-Ghaithi, ${ }^{1}$ John Husband, ${ }^{2}$ Sultan Al-Maskari ${ }^{1}$

ABSTRACT: Objectives: Bone failure due to avascular necrosis (AVN) is a complex pathological phenomenon. Analysis of molecular changes in the bone matrix may help to shed light on the disease process and guide management. This study aimed to explore changes in bone quality and structural damage caused by sickle cell disease (SCD)induced AVN using Raman spectroscopy. Methods: A total of 10 necrotic femoral heads were obtained from seven SCD patients who underwent total hip replacements. The femoral heads were cut in half and scanned using Raman spectroscopy in correlation with preoperative magnetic resonance imaging to identify necrotic and healthy control areas. Subsequently, samples were examined to determine changes in bone mineralisation, crystallinity, carbonate content, collagen cross-linking and mineral and collagen fibril orientation. Results: Significant changes were observed in bone mineral content, mineral-to-organic content and collagen fibril orientation in necrotic compared to control areas $(P \leq 0.050)$. Conclusion: The necrotic samples displayed severe structural damage and loss of mineral and organic contents. Similar Raman signals have been reported in other metabolic bone diseases such as osteoporosis, thereby potentially supporting the use of medical treatment in AVN to promote bone quality.

Keywords: Sickle Cell Disease; Osteonecrosis; Femur Head Necrosis; Bone Mineralization; Bone Density; Bone Remodeling; Extracellular Matrix; Raman Spectroscopy.

\section{AdVANCES IN KNOWLEDGE}

This study found that sickle cell disease-induced avascular necrosis (AVN) in femoral head samples resulted in significant alterations in bone mineral type and content as well as disruption of the orientation and cross-linking of collagen fibrils in the extracellular matrix.

These ultrastructural changes were similar to those previously reported in other metabolic diseases, including animal models of osteoporosis, and may therefore explain the sporadic success of bisphosphonates in the treatment of early-stage AVN.

\section{Application to Patient Care}

These findings appear to support the use of medical therapy in patients with early-stage AVN, thereby helping to prevent further bone deterioration or even potentially improving bone quality.

\section{$\mathrm{T}$} he pathogenesis of avascular necrosis $(\mathrm{AVN})$ is complex and may arise due to multiple aetiological factors. ${ }^{1}$ Bone is a metabolically active organ composed of cells, the extracellular matrix and inorganic molecules. Overall, $90 \%$ of the organic matrix is composed of type I collagen with small amounts of non-collagenous proteins, whereas calcium and phosphate are the main inorganic mineral components. ${ }^{1}$ Three types of cells are involved in bone remodelling: osteoclasts, osteoblasts and osteocytes. Osteoclasts are responsible for the resorption of defective bone, while osteoblasts are responsible for the deposition and mineralisation of the new matrix. ${ }^{1}$ However, disruption of the dynamic bone remodelling process can lead to the production of abnormal bone.

In particular, chronic circulatory failure can disrupt this physiological balance and induce structural matrix damage. ${ }^{2}$ In patients with sickle cell disease
(SCD), AVN can potentially result in joint incongruity and physical dysfunction, eventually requiring prosthetic joint replacement surgery. ${ }^{3}$ However, the process through which circulatory failure eventually leads to the collapse of the femoral head is still not yet fully understood. Since most affected patients are young, there is an urgent need to save the femoral head in the early stages of the disease, prior to joint collapse. $^{3}$

As a result, less invasive treatment modalities have been proposed including bisphosphonates, teriparatide, vasodilators and anticoagulants. ${ }^{4}$ In particular, alendronate has been effective in the treatment of adult early-stage femoral head AVN. ${ }^{5-7}$ Vasoactive agents can improve blood flow in the terminal vessels, resulting in improved clinical and radiological outcomes. ${ }^{8}$ Similarly, anticoagulants have also been shown to improve blood flow in underlying 
A
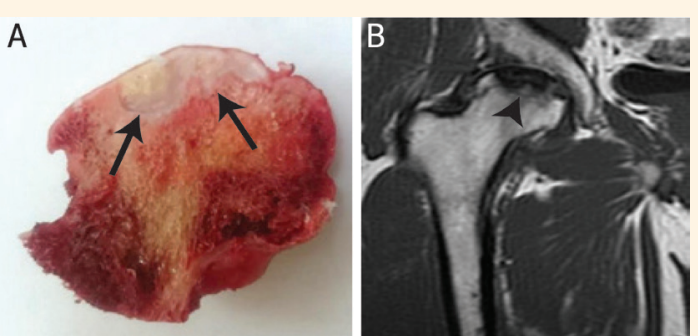

Figure 1: A: Photograph of the bisected femoral head of a patient with sickle cell disease-induced avascular necrosis showing necrosis in the subchondral area (arrow). B: Preoperative magnetic resonance imaging showing the same necrotic area as a hypointense signal (arrowhead).

coagulopathy disorders.,10 This may indicate the importance of the inhibition of platelet aggregation, while measuring pathological progression may lead to a better understanding of the effectiveness of medical therapy in treating femoral head AVN in the early stages.

Raman spectroscopy is a powerful spectroscopic tool which can be utilised to examine bone samples at the ultrastructural and molecular level based on the inelastic scattering of light by vibrating molecules. Recent research has illustrated the effectiveness of Raman spectroscopy in evaluating bone quality by providing data regarding mineral-to-matrix ratios, mineral crystallinity, carbonate content, collagen cross-linking and mineral and collagen fibril depolarisation. ${ }^{11}$ The objective of this study was to examine molecular structural changes using Raman spectroscopy in necrotic compared to normal bone samples from patients with SCD-induced AVN.

\section{Methods}

A total of 10 femoral heads were collected from seven SCD patients undergoing total hip arthroplasties for stage 5 or 6 femoral head AVN according to the
Steinberg classification system. ${ }^{12}$ The femoral heads were stored under aseptic conditions at $-20^{\circ} \mathrm{C}$ until processing. Each sample was cut into two halves and preoperative magnetic resonance imaging was used to identify necrotic and healthy control areas [Figure 1].

Raman spectroscopy results for both necrotic and normal control areas of the femoral heads were recorded and compared at 400-2,000 $\mathrm{cm}^{-1} \cdot{ }^{11}$ Each sample was scanned using a Raman spectrometer (i-Raman ${ }^{\circledR}$ EX Portable Raman Spectrometer, B\&W Tek Inc., Newark, Delaware USA) with excitation from a laser diode operating at 1,064 $\mathrm{nm}$. Corresponding spectroscopy software (B\&W Tek Inc.) was used to analyse the obtained bone spectra. The spectra were baselined and averaged to improve the signal-to-noise ratio. The identification of key Raman bands was performed manually.

Each sample was assessed for degree of bone mineralisation, mineral crystallinity, carbonate content, collagen cross-linking and mineral and collagen fibril orientation. Ratios of mineral content and mineral-toorganic matrix content were generated for analytical purposes. The volume of bone mineral content was assessed by comparing phosphate and carbonate bands at 958 and $1,070 \mathrm{~cm}^{-1}$, respectively, relative to that of the amide band at $1,656 \mathrm{~cm}^{-1}$. Bone mineral crystallinity was studied by comparing the carbonate band at $1,070 \mathrm{~cm}^{-1}$ to the phosphate band at $958 \mathrm{~cm}^{-1}$. This ratio corresponds to the extent of carbonate substitution for carbonate in bone mineral crystals and is inversely related to bone remodelling. ${ }^{1}$ Collagen represents the organic scaffold providing the tensile and viscoelastic properties of bone. The collagen cross-linking ratio was examined by comparing the shift in the amide I band from 1,660 to $1,684 \mathrm{~cm}^{-1}$.

All data were entered into the Statistical Package for the Social Sciences (SPSS), Version 22 (IBM Corp., Chicago, Illinois, USA). Means and standard
A

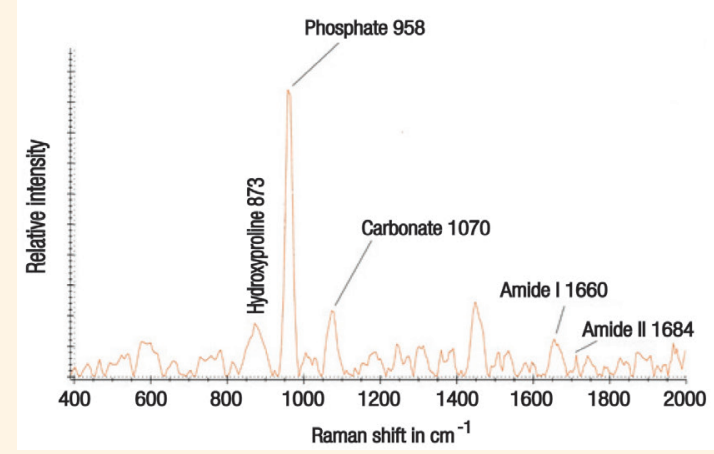

B

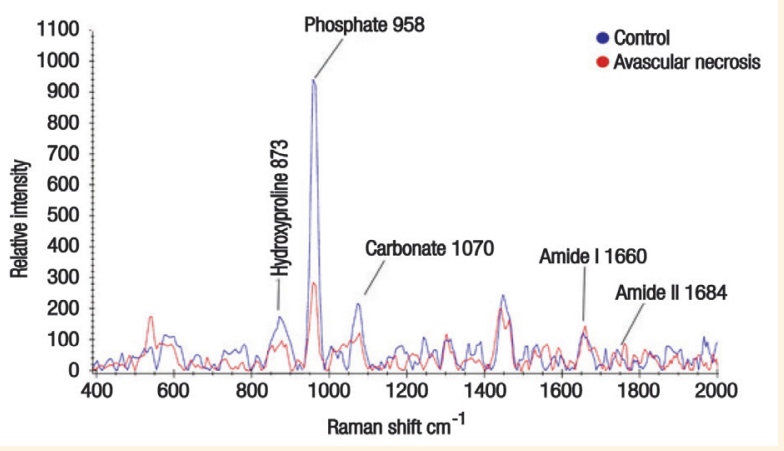

Figure 2: Graphs comparing the acquired Raman spectra of (A) normal bone and (B) bone collected from femoral heads affected by sickle cell disease-induced avascular necrosis $(\mathrm{N}=10)$. The normal bone shows peaks at $958 \mathrm{~cm}^{-1}$ for phosphate, $1,070 \mathrm{~cm}^{-1}$ for carbonate, $1,660 \mathrm{~cm}^{-1}$ for amide I and $1,684 \mathrm{~cm}^{-1}$ for amide II. It demonstrates the overall drop in spectra of necrotised bone. 


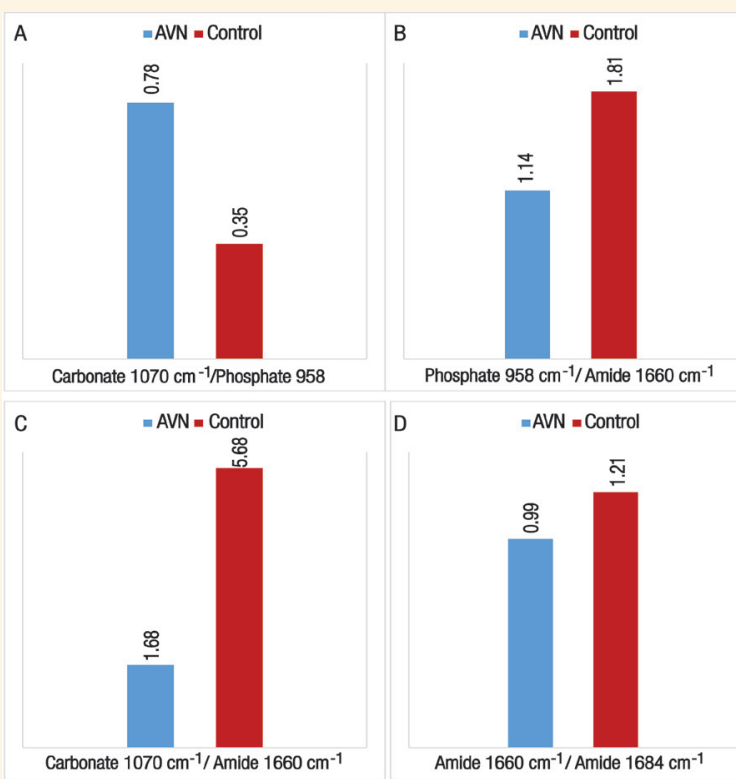

Figure 3: Charts showing the average acquired Raman spectra from bone samples collected from femoral heads affected by sickle cell disease-induced avascular necrosis $(\mathrm{N}=10)$. In comparison to healthy control areas, the necrotic areas showed (A) an increased carbonate-to-phosphate ratio, indicating a difference in bone crystallinity and transformation to a brittle matrix, $(\mathbf{B} \& \mathbf{C})$ reduced phosphate-to-amide and carbonate-to-amide ratios, indicating a reduction in mineral relative to organic material, and (D) a reduced collagen crosslinking ratio, indicating increased collagen cross-linkage disruption.

$A V N=$ avascular necrosis.

Table 1: Average ratios of matrix and minerals based on acquired Raman spectra from bone samples collected from femoral heads affected by sickle cell disease-induced avascular necrosis $(\mathrm{N}=10)$

\begin{tabular}{|c|c|c|c|c|c|c|c|c|}
\hline \multirow[t]{2}{*}{ Sample } & \multicolumn{2}{|c|}{$\begin{array}{l}\text { Carbonate at } 1,070 \mathrm{~cm}^{-1} \\
\text { to phosphate at } 958 \mathrm{~cm}^{-1}\end{array}$} & \multicolumn{2}{|c|}{$\begin{array}{l}\text { Carbonate at } 1,070 \mathrm{~cm}^{-1} \\
\text { to amide } I \text { at } 1,660 \mathrm{~cm}^{-1}\end{array}$} & \multicolumn{2}{|c|}{$\begin{array}{l}\text { Phosphate at } 958 \mathrm{~cm}^{-1} \\
\text { to amide } \mathrm{I} \text { at } 1,660 \mathrm{~cm}^{-1}\end{array}$} & \multicolumn{2}{|c|}{$\begin{array}{l}\text { Amide I at } 1,660 \mathrm{~cm}^{-1} \text { to } \\
\text { amide II at } 1,684 \mathrm{~cm}^{-1}\end{array}$} \\
\hline & AVN & Control & AVN & Control & AVN & Control & AVN & Control \\
\hline 1 & 0.58 & 0.30 & 1.92 & 8.74 & 1.11 & 2.64 & 0.92 & 0.83 \\
\hline 2 & 0.78 & 0.43 & 1.67 & 2.57 & 1.30 & 1.09 & 0.91 & 1.06 \\
\hline 3 & 0.93 & 0.43 & 1.39 & 3.88 & 1.29 & 1.67 & 0.96 & 1.08 \\
\hline 4 & 0.88 & 0.42 & 1.47 & 3.96 & 1.29 & 1.67 & 1.03 & 1.27 \\
\hline 5 & 0.80 & 0.29 & 1.92 & 2.31 & 1.53 & 0.67 & 0.81 & 1.44 \\
\hline 6 & 0.98 & 0.20 & 1.33 & 1.89 & 1.30 & 0.38 & 1.03 & 1.04 \\
\hline 7 & 0.87 & 0.43 & 1.64 & 3.88 & 1.43 & 1.67 & 1.54 & 1.08 \\
\hline 8 & 0.85 & 0.22 & 1.29 & 9.54 & 1.10 & 2.09 & 0.90 & 1.34 \\
\hline 9 & 0.58 & 0.49 & 2.15 & 4.06 & 1.24 & 2.00 & 0.99 & 1.87 \\
\hline 10 & 0.55 & 0.27 & 2.03 & 15.84 & 1.11 & 4.22 & 0.84 & 1.04 \\
\hline Average & $0.78 \pm 0.32$ & $0.35 \pm 0.05$ & $1.68 \pm 0.44$ & $5.68 \pm 1.39$ & $1.14 \pm 0.09$ & $1.81 \pm 0.34$ & $0.99 \pm 0.65$ & $1.21 \pm 0.31$ \\
\hline
\end{tabular}

AVN = atraumatic avascular necrosis

deviations were examined using a Student's t-test and analysis of variance. The level of statistical significance was set at $P \leq 0.050$. This study was approved by the Medical Research \& Ethics Committee of the College of Medicine \& Health Sciences at Sultan Qaboos University (SQU-EC1039114). Written informed consent was obtained from all patients prior to surgery.
Consent of the patient was obtained for publication of Figure 1.

\section{Results}

Of the seven patients with SCD included in the study, five $(71.4 \%)$ were female and three (42.9\%) underwent 
a staged bilateral arthroplasty. The average age was 25 years (range: $22-32$ years). Overall, AVN resulted in a drop in bone quality as measured by the mineral-tomatrix ratio, mineral crystallinity, carbonate content, collagen cross-linking ratio and orientation of the mineral and collagen fibrils.

Figure 2 shows the average collected spectra of necrotic compared to healthy bone, along with the band assignment. There was a significant decrease in both phosphate-to-amide ratio $(P=0.01$; mean ratio of $1.14 \pm 0.09$ from necrotic areas and $1.81 \pm$ 0.34 from healthy area) and carbonate-to-amide ratio $(P=0.02$; mean ratio of $1.68 \pm 0.44$ from necrotic areas and $5.68 \pm 1.39$ from healthy area) observed for necrotic compared to control areas, indicating a drop in mineral volume. In addition, there was a significant drop in the carbonate-to-phosphate ratio for the necrotic compared to control areas $(P=0.008$; mean ratio of $1.14 \pm 0.09$ from necrotic areas and $1.81 \pm 0.34$ from healthy area) and significant shift from amide signal at $1,660 \mathrm{~cm}^{-1}$ to amide at $1,684 \mathrm{~cm}^{-1}$ (mean ratio of $0.99 \pm 0.65$ from necrotic areas and $1.21 \pm 0.31$ from healthy area) [Table 1]. Figure 3 demonstrates the drop of the overall mineral-to-matrix ratios in the necrotic compared to the control.

\section{Discussion}

The current study aimed to examine molecular structural changes in necrotic compared to normal bone samples from patients with SCD-induced AVN using Raman spectroscopy. The results indicated that SCDinduced AVN resulted in severe structural damage to the bone and a loss of mineral and organic contents, with necrotic areas showing a significant loss in bone quality and protein formation compared with the healthy control areas. These changes in bone quality were apparent even at an advanced stage of disease.

These results are consistent with the concept of bone remodelling disruption due to circulatory failure, in which the bone loses its ability to remodel due to disturbances in bone tissue vascularity. ${ }^{13}$ Low levels of oxygen and nutrition disrupt the physiological bone remodelling process, leading to the production of low-quality bone. In addition, the shift of collagen spectra from 1,660 to $1,684 \mathrm{~cm}^{-1}$ suggests the disruption of collagen cross-linking, which would lead to the deformation of the fibril scaffold, loss of tensile strength and eventually bone collapse. Silva et al. reported similar findings in a senescence-accelerated mouse prone 6 model of osteoporosis due to reduced collagen content and collagen-to-mineral ratios. ${ }^{14}$
Bone mineralisation is the process by which mineral crystals are deposited in the organic bone matrix, providing structural strength; however, any alterations to this process can result in the bone having poor biomechanical properties. ${ }^{1}$ Raman spectrometry can determine the degree of bone mineralisation by measuring different ratios including the mineralto-matrix ratio, carbonate-to-phosphate ratio and degree of mineral crystallinity. ${ }^{11}$ The latter variable is attributed to the altered bone remodelling process which transforms the normal bone into a more brittle tissue susceptible to fragility fractures.

In the present study, the results indicated a significant reduction of mineral-to-matrix ratios in necrotic compared to control areas, as represented by the ratios of phosphate at $958 \mathrm{~cm}^{-1}$ or carbonate at $1,070 \mathrm{~cm}^{-1}$ relative to that for collagen amide I at $1,656 \mathrm{~cm}^{-1}$. Moreover, there was a significant increase in the carbonate-to-phosphate ratio in the necrotic compared to control areas, with the ratio increasing with reduced remodelling. Acquired Raman signals from the necrotic bone samples were comparable to those reported from other bone pathologies known to respond to antiresorptive therapy such as osteoporosis. $^{15,16}$ For example, a study of cancellous bone collected from the lumbar spine of female cadavers indicated a reduced mineral-to-matrix ratio in those with osteoporosis. ${ }^{15}$ Similarly, Shen et al. observed a decrease in the mineral-to-matrix ratios of rats with spinal cord injury-induced osteoporosis, a finding attributed to hormonal changes. ${ }^{16}$

In $\mathrm{AVN}$, the rationale behind the use of medical therapy such as bisphosphonates is that bone collapse may be prevented during the repair phase by the inhibition or slowing down of resorptive activity. ${ }^{17}$ Using Raman spectroscopy, various studies have shown that the response of osteoporotic bone to antiresorptive agents leads to improved mineral and matrix ratios. ${ }^{18,19}$ Burket et al. found that the femurs of osteoporotic sheep treated with antiresorptive agents demonstrated improved parameters in terms of carbonate substitution, crystallinity and collagen. ${ }^{18}$ Anabolic agents such as teriparatide, a form of parathyroid hormone 1-34, have also been shown to have a positive in vivo time-dependent effect on bone mineral and matrix quality. ${ }^{19}$ In line with previous research, the findings of the present study suggest that Raman spectroscopy may be useful in studying the effect of these agents in animal or even human models. However, further work is advocated, especially in the early stages of disease, in order to better understand the disease process and help develop timely therapeutic strategies. 


\section{Conclusion}

The current study found that necrotic areas of bone collected from the femoral heads of SCD patients with AVN demonstrated abnormal structural features compared to normal bone, thereby indicating poorer mechanical properties. However, acquired Raman signals from these necrotic areas were similar to those reported in other metabolic diseases found to respond well to medical treatment such as osteoporosis. Such findings appear to support the use of medical therapy in the early stages of AVN, as well as highlight the utility of Raman spectroscopy in studying the physiological bone remodelling process.

\section{CONFLICT OF INTEREST}

The authors declare no conflicts of interest.

\section{FUNDING}

This study was funded by The Research Council, Oman, through the 2016 Individual Innovation Competition Scheme.

\section{References}

1. Clarke B. Normal bone anatomy and physiology. Clin J Am Soc Nephrol 2008; 3:S131-9. https://doi.org/10.2215/CJN.04151206.

2. Boettcher WG, Bonfiglio M, Hamilton HH, Sheets RF, Smith K. Non-traumatic necrosis of the femoral head: I. Relation of altered hemostasis to etiology. J Bone Joint Surg Am 1970; 52:312-21. https://doi.org/10.2106/00004623-197052020-00012.

3. Vaishya R, Agarwal AK, Edomwonyi EO, Vijay V. Musculoskeletal manifestations of sickle cell disease: A review. Cureus 2015; 7:e358. https://doi.org/10.7759/cureus.358.

4. Marker DR, Seyler TM, McGrath MS, Delanois RE, Ulrich SD Mont MA. Treatment of early stage osteonecrosis of the femoral head. J Bone Joint Surg Am 2008; 90:175-87. https://doi.org/10.2 106/JBJS.H.00671.

5. Agarwala S, Jain D, Joshi VR, Sule A. Efficacy of alendronate, a bisphosphonate, in the treatment of AVN of the hip: A prospective open-label study. Rheumatology (Oxford) 2005; 44:352-9. https://doi.org/10.1093/rheumatology/keh481

6. Lai KA, Shen WJ, Yang CY, Shao CJ, Hsu JT, Lin RM. The use of alendronate to prevent early collapse of the femoral head in patients with nontraumatic osteonecrosis: A randomized clinical study. J Bone Joint Surg Am 2005; 87:2155-9. https:// doi.org/10.2106/JBJS.D.02959.
7. Luo RB, Lin T, Zhong HM, Yan SG, Wang JA. Evidence for using alendronate to treat adult avascular necrosis of the femoral head: A systematic review. Med Sci Monit 2014; 20:2439-47. https://doi.org/10.12659/MSM.891123.

8. Jäger M, Tillmann FP, Thornhill TS, Mahmoudi M, Blondin D, Hetzel GR, et al. Rationale for prostaglandin I2 in bone marrow oedema: From theory to application. Arthritis Res Ther 2008. 10:R120. https://doi.org/10.1186/ar2526.

9. Glueck CJ, Freiberg RA, Sieve L, Wang P. Enoxaparin prevents progression of stages I and II osteonecrosis of the hip. Clin Orthop Relat Res 2005; 435:164-70. https://doi.org/10.1097/01. blo.0000157539.67567.03

10. Johnson AJ, Mont MA, Tsao AK, Jones LC. Treatment of femoral head osteonecrosis in the United States: 16-year analysis of the Nationwide Inpatient Sample. Clin Orthop Relat Res 2014; 472:617-23. https://doi.org/10.1007/s11999-013-3220-3.

11. Morris MD, Mandair GS. Raman assessment of bone quality. Clin Orthop Relat Res 2011; 469:2160-9. https://doi.org/10.10 07/s11999-010-1692-y.

12. Steinberg ME, Steinberg DR. Classification systems for osteonecrosis: An overview. Orthop Clin North Am 2004; 35:273-83. https://doi.org/10.1016/j.ocl.2004.02.005.

13. Parfitt AM, Mundy GR, Roodman GD, Hughes DE, Boyce BF. A new model for the regulation of bone resorption, with particular reference to the effects of bisphosphonates. J Bone Miner Res 1996; 11:150-9. https://doi.org/10.1002/jbmr.5650110203.

14. Silva MJ, Brodt MD, Wopenka B, Thomopoulos S, Williams D, Wassen $\mathrm{MH}$, et al. Decreased collagen organization and content are associated with reduced strength of demineralized and intact bone in the SAMP6 mouse. J Bone Miner Res 2006; 21:78-88. https://doi.org/10.1359/JBMR.050909.

15. Kim G, Cole JH, Boskey AL, Baker SP, van der Meulen MC Reduced tissue-level stiffness and mineralization in osteoporotic cancellous bone. Calcif Tissue Int 2014; 95:125-31. https://doi. org/10.1007/s00223-014-9873-4.

16. Shen J, Fan L, Yang J, Shen AG, Hu JM. A longitudinal Raman microspectroscopic study of osteoporosis induced by spinal cord injury. Osteoporos Int 2010; 21:81-7. https://doi.org/10.10 07/s00198-009-0949-3

17. Young ML, Little DG, Kim HK. Evidence for using bisphosphonate to treat Legg-Calvé-Perthes disease. Clin Orthop Relat Res 2012; 470:2462-75. https://doi.org/10.1007/s11999-011-2240-0.

18. Burket JC, Brooks DJ, MacLeay JM, Baker SP, Boskey AL, van der Meulen $\mathrm{MCH}$. Variations in nanomechanical properties and tissue composition within trabeculae from an ovine model of osteoporosis and treatment. Bone 2013; 52:326-36. https:// doi.org/10.1016/j.bone.2012.10.018.

19. Pettway GJ, Schneider A, Koh AJ, Widjaja E, Morris MD, Meganck JA, et al. Anabolic actions of PTH (1-34): Use of a novel tissue engineering model to investigate temporal effects on bone. Bone 2005; 36:959-70. https://doi.org/10.1016/j. bone.2005.02.015 